\title{
Solutions nodales sur les variétés Riemanniennes non locale- ment conformément plates à bord
}

\author{
David Holcman
}

\begin{abstract}
Dans cet article, on considère le problème de Dirichlet pour une EDP non linéaire avec une donnée au bord qui change de signe. L'existence d'une famille de solutions minimisantes est obtenue sur les variétés riemaniennes compactes non localement conformément plates à bord.

This paper deals with solutions of Dirichlet problem for a nonlinear PDE with boundary data changing of sign. A family of minimizing nodal solutions are found on nonlocally conformally flat manifolds with boundary.
\end{abstract}

Mathematics Subject Classification (2000). 53C21, 58J05.

Keywords. Nonlinear PDE, critical exponent, Riemannian manifolds, boundary value problem, variational methods.

\section{Introduction}

Soit $\left(\mathrm{V}_{n}, g\right)$ une variété riemannienne compacte à bord $\mathrm{C}^{\infty}$, de dimension $n \geq$ 3 . Soient $a$ et $f$ deux fonctions dans $\mathrm{C}^{\infty}\left(\mathrm{V}_{n}\right)$ et $\phi$ une une fonction dans $\mathrm{C}^{\infty}\left(\partial \mathrm{V}_{n}\right)$. La fonction $\mathrm{f}$ est supposée positive sur $\mathrm{V}_{n}$.

On étudie l'existence d'un réel positif $\lambda$ et d'une solution minimisante $u$ de l'équation

$$
\begin{aligned}
\Delta_{g} u+a u & = & \lambda f|u|^{\frac{4}{n-2}} u & & \text { sur } \mathrm{V}_{n} \\
u & = & \phi & & \text { sur } \partial \mathrm{V}_{n}
\end{aligned}
$$

L'équation étudiée contient l'exposant critique, dans l'inclusion de Sobolev. Une large famille de problèmes est dédiée à ce type d'étude, nous allons en rappeler quelques-uns. Le résultat de cet article est énoncé dans le théorème (1) et il est à noter que la solution obtenue minimise une fonctionnelle d'énergie.

Rappelons qu'une solution non minimisante a été obtenue par une analyse élémentaire lorsque la donnée au bord $\phi$ n'est pas identiquement nulle: il a été montré [8] que pour tout $\epsilon>0$, il existe un réel $\lambda_{\epsilon} \in(0, \epsilon)$ et une fonction $u_{\epsilon}$ 
de classe $\mathrm{C}^{2}$ tels que

$$
\begin{aligned}
& \Delta_{g} u_{\epsilon}+a u_{\epsilon}=f\left|u_{\epsilon}\right|^{\frac{4}{n-2}} u_{\epsilon} \quad \text { sur } \mathrm{V}_{n} \\
& u_{\epsilon}=\quad \lambda_{\epsilon} \phi \quad \text { sur } \partial \mathrm{V}_{n}
\end{aligned}
$$

La preuve est donnée lorsque $\mathrm{V}_{n}$ est un ouvert borné $\Omega$ de $\mathbb{R}^{n}$ et $g$ une métrique Riemannienne définie dans un voisinage de $\bar{\Omega}$. Dans ce travail, rien ne dit que la solution soit le minimum d'une fonctionnelle d'énergie.

Le résultat complète aussi celui obtenu par Caffarelli-Spruck [5] qui se limite aux ouverts à bord de $\mathbb{R}^{n}$ lorsque $\mathrm{f}$ est constante et la donnée au bord $\phi$ est positive. L'ingrédient essentiel de la preuve est la méthode de réflexion [6]. Ici on montre l'existence d'une famille de solutions minimisantes pour une classe assez vaste de fonctions régulières $f$. Le résultat présenté ici généralise aussi celui obtenu par Brezis [4], sur les ouverts bornés de $\mathbb{R}^{n}$.

Ce travail a été annoncé dans [9] et l'originalité réside dans l'analyse du possible phénomène de concentration. Les conditions mises en évidence dans la proposition 1 sont nouvelles, généralisant celle sur le minimum de la fonctionnelle de Yamabe [1]. Cela permet de trouver des solutions minimisantes de (1) sous des conditions géométriques. On peut dire, sous les hypothèses du théorème (1), que la condition géométrique sur le tenseur de Weyl l'emporte sur la donnée au bord non nulle pour obtenir les solutions minimisantes.

Théorème 1. Soit $\left(\mathrm{V}_{n}, g\right)$ une variété Riemannienne compacte à bord, de courbure scalaire $\mathrm{R}$ sur laquelle on considère l'équation (1). On suppose que la première valeur propre de l'opérateur $\left(\Delta_{g}+a\right)$ est positive et que $f$ atteint un de ses maxima en un point intérieur $P$, pour lequel $\Delta_{g} f(\mathrm{P})=0$. Si l'une des deux conditions suivantes est vérifiée

- $\quad a=\frac{n-2}{4(n-1)} \mathrm{R}, \operatorname{dim} \mathrm{V}_{n}=n>6, \Delta_{g}^{2} f(\mathrm{P})=0$ et le tenseur de Weyl ne s'annule pas en $P$ (ce qui exclut les ouverts de $\mathbb{R}^{n}$ standard );

- $\quad a \neq \frac{n-2}{4(n-1)} \mathrm{R}, \operatorname{dim} \mathrm{V}_{n}=n>4$ et $a(\mathrm{P})<\frac{n-2}{4(n-1)} \mathrm{R}(\mathrm{P})$;

alors il existe un réel positif $\gamma_{0}$, dépendant de $\phi$, une famille de nombre réels $\left(\lambda_{p, \gamma}\right)_{\gamma>\gamma_{0}}$ et une famille de fonctions $\left(u_{\gamma}\right)_{\gamma>\gamma_{0}}$ telles que pour tout $\gamma>\gamma_{0}$

1. $\lambda_{p, \gamma}$ et $u_{\gamma}$ sont solutions de l'équation (1)

2. $u_{\gamma}$ est une solution minimisante de la fonctionelle I définie ci-dessous:

La fonctionnelle I est définie sur $\stackrel{\circ}{H}{ }_{1}\left(\mathrm{~V}_{n}\right)$ par $\mathrm{I}(u)=\int_{\mathrm{V}_{n}}|\nabla u|^{2}+a u^{2}$ sous la contrainte $\int_{\mathrm{V}_{n}} f|u+h|^{2 n /(n-2)}=\gamma$ où $h$ est l'unique solution de l'équation

$$
\begin{array}{r}
\Delta_{g} h+a h=0 \text { sur } \mathrm{V}_{n} \\
h=\phi \text { sur } \partial \mathrm{V}_{n}
\end{array}
$$

En particulier, la solution $u_{\gamma}$ est nodale si la donnée au bord $\phi$ change de signe. 
Remarque. Les conditions données en hypothèse lorsque $a=\frac{n-2}{4(n-1)} \mathrm{R}$ ne dépendent que de la structure conforme de la variété. On remarquera également qu'il n'est pas nécessaire que la donnée au bord $\phi$ ne soit pas nulle pour obtenir un théorème similaire: dans le cas ou $\phi$ est identiquement nulle, des résultats sont connus, voir [1].

Le plan de la démonstration du théorème 1 est le suivant: dans le paragraphe 2 , on construit par minimisation une suite de solutions aux équations du type (1) avec exposant sous-critique. Le paragraphe 3 donne le comportement possible de cette suite de solutions minimisantes lorsque l'exposant sous-critique tend vers l'exposant critique. Le paragraphe 4 met en évidence des conditions dites de non-concentration. Enfin la dernière partie est consacrée au phénomène de concentration. Des conditions sur la fonction $f$ et sur la géométrie conforme sont introduites pour empêcher l'existence de points de concentration.

\section{Construction de solutions sous-critiques}

Dans cette partie, nous allons considérer l'équation (1) avec un second membre de la forme $\lambda f|u|^{p-2} u$, et $\mathrm{p}$ sous-critique c'est-à-dire $p<N$, où l'on a noté $N=\frac{2 n}{n-2}$.

Dans le but de transformer l'équation (1), nous commençons par étendre la donnée au bord $\phi$ par l'unique fonction régulière $h$ définie $\mathrm{V}_{n}$ par

$$
\Delta_{g} h+a h=0 \text { sur } \mathrm{V}_{n} \text { et } h=\phi \text { sur } \partial \mathrm{V}_{n}
$$

dont l'existence est assurée par la coercivité de l'opérateur $\left(\Delta_{g}+a\right)$. Construisons maintenant la solution avec exposant sous-critique. Nous supposons ici que $a=$ $\frac{n-2}{4(n-1)} \mathrm{R}=\overline{\mathrm{R}}$ et considérons la fonctionnelle d'énergie I définie par

$$
\mathrm{I}(u)=\int_{\mathrm{V}_{n}}\left(|\nabla u|^{2}+\overline{\mathrm{R}} u^{2}\right)
$$

Soit $\mathrm{A}_{p, \gamma}=\left\{u \in \stackrel{o}{H}_{1}\left(\mathrm{~V}_{n}\right)\left|\int f\right| u+\left.h\right|^{p}=\gamma\right\}$ où $p<N$ est voisin de $N$ et $h$ a été définie dans le paragraphe précédent.

Définissons enfin le minimum de la fonctionnelle d'énergie $\mathrm{I}(u)$,

$$
\mu_{p, \gamma}=\inf _{\mathrm{A}_{p, \gamma}} \mathrm{I}(u)=\inf _{\mathrm{A}_{p, \gamma}} \int_{\mathrm{V}_{n}}\left(|\nabla u|^{2}+\overline{\mathrm{R}} u^{2}\right)
$$

Comme l'opérateur $\Delta+\overline{\mathrm{R}}$ est coercif, $\mu_{p, \gamma}$ est positif.

Vérifions maintenant que si la condition suivante

$$
\int_{\mathrm{V}_{n}} f|h|^{N}<\gamma
$$

est satisfaite alors $\mathrm{A}_{p, \gamma}$ est un ensemble non vide. On supposera d'ailleurs dans la suite que cette condition sur $\gamma$ est toujours vérifiée. Pour montrer que $\mathrm{A}_{p, \gamma}$ 
est non vide, soit $\phi_{1}$ une fonction propre associée à la première valeur propre $\lambda_{1}$ du Laplacien définie par:

$$
\begin{aligned}
& \Delta \phi_{1}=\lambda_{1} \phi_{1} \quad \text { sur } \mathrm{V}_{n} \\
& \phi_{1}=\quad 0 \quad \text { sur } \partial \mathrm{V}_{n}
\end{aligned}
$$

La fonction continue $\mathrm{F}(t)=\int_{\mathrm{V}_{n}} f\left|t \phi_{1}+h\right|^{p}$ vérifie

- $\lim _{t \rightarrow+\infty} \mathrm{F}(t)=\infty$

- $\mathrm{F}(0)=\int_{\mathrm{V}_{n}} f|h|^{N}<\gamma$

Ainsi il existe un réel $t_{p, \gamma}$ tel que $\mathrm{F}\left(t_{p, \gamma}\right)=\int_{\mathrm{V}_{n}} f\left|t_{p, \gamma} \phi_{1}+h\right|^{p}=\gamma$. Ce qui prouve que la fonction $t_{p, \gamma} \phi_{1} \in \mathrm{A}_{p, \gamma}$.

Montrons maintenant qu'il existe une fonction $w_{p, \gamma}$ telle que $\mathrm{I}\left(w_{p, \gamma}\right)=\mu_{p, \gamma}$. Pour cela soit $w_{k}$ une suite de fonctions dans $\mathrm{A}_{p, \gamma}$ minimisante pour I. Cette suite est bornée dans $\stackrel{o}{H_{1}}$ (on utilise l'inégalité de Poincaré pour majorer la norme $\mathrm{L}_{2}$ ). On peut donc en extraire une sous-suite qui converge faiblement dans $\stackrel{o}{H_{1}}$, fortement dans $\mathrm{L}_{p}$ avec $p<N$ et presque partout sur $\mathrm{V}_{n}$ vers une fonction $w_{p, \gamma}$, voir [1].

Le calcul des variations donne alors l'existence d'un multiplicateur de Lagrange $\lambda_{p, \gamma}$ tel que la limite $w_{p, \gamma}$ vérifie faiblement,

$$
\begin{aligned}
\Delta w_{p, \gamma}+\overline{\mathrm{R}} w_{p, \gamma} & =\lambda_{p, \gamma} f\left|w_{p, \gamma}+h\right|^{p-2}\left(w_{p, \gamma}+h\right) \operatorname{sur} \mathrm{V}_{n} \\
w_{p, \gamma} & =0 \text { sur } \partial \mathrm{V}_{n}
\end{aligned}
$$

Enfin comme $\left|w_{p, \gamma}+h\right|^{p-2}\left(w_{p, \gamma}+h\right)$ est dans $\mathrm{C}^{1, \alpha}\left(\mathrm{V}_{n}\right)$ les théorèmes de régularité montrent que $\left|w_{p, \gamma}+h\right|^{p-2}\left(w_{p, \gamma}+h\right)$ est dans $\mathrm{C}^{3, \alpha}\left(\mathrm{V}_{n}\right)$, (voir [7]).

\section{Convergence vers une solution}

Dans cette partie, on étudie le comportement de la suite $w_{p, \gamma}$ lorsque p tend vers l'exposant critique $N$. Nous commençons par montrer deux lemmes,

Lemme 1. Lorsque la condition (8) est vérifiée, $\gamma$ étant fixé, le multiplicateur de Lagrange $\lambda_{p, \gamma}$ est positif $\lambda_{p, \gamma}>0$ et la suite $\left(\lambda_{p, \gamma}\right)$ pour la variable $p$ est bornée. De plus la suite $\left(w_{p, \gamma}\right)$ est bornée dans $H_{1}$.

Preuve. Comme $\lim _{p \rightarrow N} \int_{\mathrm{V}_{n}} f|h|^{p}<\gamma$, l'inégalité stricte reste vraie pour $\mathrm{p}$ proche de N. Ainsi en multipliant l'équation (10) par $w_{p, \gamma}$ et en utilisant la définition du minimum d'énergie $\mu_{p, \gamma}$ :

$$
\begin{gathered}
0 \leq \mu_{p, \gamma}=\int\left|\nabla w_{p, \gamma}\right|^{2}+\overline{\mathrm{R}} w_{p, \gamma}^{2}= \\
\lambda_{p, \gamma}\left(\gamma-\int f\left|w_{p, \gamma}+h\right|^{p-2}\left(w_{p, \gamma}+h\right) h\right)
\end{gathered}
$$


Or pour p voisin de N, l'inégalité de Hölder donne

$$
\int f\left|w_{p, \gamma}+h\right|^{p-2}\left(w_{p, \gamma}+h\right) h \leq \gamma^{1-1 / p}\left(\int f|h|^{p}\right)^{1 / p}<\gamma
$$

Comme $\mu_{p, \gamma} \geq 0$, on en déduit que $\lambda_{p, \gamma} \geq 0$. Si $\lambda_{p, \gamma}=0$ alors $w_{p, \gamma}=0$, ce qui provient de l'équation (10) et de la condition (8). On en déduit une contradiction avec le fait que $w_{p, \gamma} \in \mathrm{A}_{p, \gamma}$. Montrons maintenant que la suite $\lambda_{p, \gamma}$ est bornée. En utilisant la fonction ayant servi à montrer que $\mathrm{A}_{p, \gamma}$ est non vide, on obtient la majoration

$$
\int\left|\nabla w_{p, \gamma}\right|^{2}+\overline{\mathrm{R}} w_{p, \gamma}^{2} \leq \mathrm{I}\left(t_{p, \gamma} \phi_{1}\right) \leq \mathrm{C} t_{p, \gamma}^{2}
$$

où $\mathrm{C}$ est une constante indépendante de $p$ et $\gamma$. Fixons $\gamma$, comme $t_{p, \gamma} \phi_{1} \in \mathrm{A}_{p, \gamma}$ on a $\int_{\mathrm{V}_{n}} f\left|t_{p, \gamma} \phi_{1}+h\right|^{p}=\gamma$ et $\int_{\mathrm{V}_{n}} f\left|\phi_{1}\right|^{N}>0$. On en déduit que la suite $\left(t_{p, \gamma}\right)$ ne peut pas tendre vers l'infini quand p tend vers $N$. La suite $\left(t_{\underline{p}, \gamma}\right)$ est donc bornée quand $\mathrm{p}$ tend vers $\mathrm{N}$. Maintenant comme l'opérateur $\Delta+\overline{\mathrm{R}}$ est coercif, on en déduit que la suite $\left(w_{p, \gamma}\right)$ est bornée dans $H_{1}$ quand $p$ tend vers $N$. En combinant alors (11) et (12), on obtient la majoration:

$$
0<\lambda_{p, \gamma} \leq \frac{\mathrm{C}(\gamma)}{\gamma-\gamma^{1-1 / p}\left(\int f|h|^{p}\right)^{1 / p}} \leq \mathrm{C}^{\prime}(\gamma, h)
$$

Ce qui prouve que la suite $\left(\lambda_{p, \gamma}\right)$ est bornée quand $p$ tend vers $N$. Pour $\gamma$ fixé on obtient donc une borne dans $H_{1}\left(\mathrm{~V}_{n}\right)$ pour la suite $\left(w_{p, \gamma}\right)$ lorsque p tend vers $\mathrm{N}$. Ce lemme 1 nous permet alors d'extraire une sous-suite $\left(w_{p, \gamma}\right)$ telle que

- $\left\|w_{p, \gamma}\right\|_{H_{1}} \leq \mathrm{C}$

- $w_{p, \gamma} \rightarrow w_{0}$ dans $\stackrel{o}{H_{1}}$ faiblement

- $w_{p, \gamma} \rightarrow w_{0}$ dans $\mathrm{L}_{r}\left(\mathrm{~V}_{n}\right)$ fortement où $r=\sup (2, N-1)$

- $w_{p, \gamma} \rightarrow w_{0}$ presque partout sur $\mathrm{V}_{n}$

Lemme 2. Pour $\gamma$ assez grand, $\lambda_{p, \gamma}$ ne tend pas vers 0 quand $p$ tend vers $N$.

Preuve. Supposons que la suite $\lambda_{p, \gamma} \rightarrow 0$ quand $p \rightarrow N$, alors en utilisant l'équation (10) on en déduit que $\int\left|\nabla w_{p, \gamma}\right|^{2}+\overline{\mathrm{R}} w_{p, \gamma}^{2} \rightarrow 0$ quand $\mathrm{p}$ tend vers $\mathrm{N}$. Comme l'opérateur $\Delta+\overline{\mathrm{R}}$ est coercif, on en déduit que la suite $w_{p, \gamma}$ tend vers 0 dans $H_{1}$ quand $p$ tend vers $N$. Finalement en utilisant l'inégalité de Sobolev [1] on en déduit que $w_{p, \gamma} \rightarrow 0$ quand $p \rightarrow N$ dans $\mathrm{L}_{N}$. On en déduit donc:

$$
\int f\left|w_{p, \gamma}+h\right|^{p} \rightarrow \int f|h|^{N}
$$

quand $p \rightarrow N$. Mais comme $w_{p, \gamma} \in \mathrm{A}_{p, \gamma}, \gamma=\int f\left|w_{p, \gamma}+h\right|^{p}$,ce qui est en contradiction avec la condition (8). Finalement la suite $\lambda_{p, \gamma}$ ne tend pas vers 0 quand $p \rightarrow N$. 
Ainsi $\left(\lambda_{p, \gamma}\right)$ reste bornée et ne tend pas vers 0 si $\gamma$ est assez grand. Quitte à extraire une sous-suite, $\lambda_{p, \gamma}$ converge vers $\lambda>0$ et $\left(w_{p, \gamma}\right)$ vers $w_{0}$, une solution faible dans $\stackrel{o}{H}$ de l'équation suivante:

$$
\begin{aligned}
& \Delta w_{0}+\mathrm{R} w_{0}=\lambda f\left|w_{0}+h\right|^{4 /(n-2)}\left(w_{0}+h\right) \quad \text { sur } \mathrm{V}_{n} \\
& w_{0}=\quad 0 \quad \text { sur } \partial \mathrm{V}_{n}
\end{aligned}
$$

Comme le second membre est de classe $\mathrm{C}^{1, \alpha}$, on en déduit que $w_{0} \in \mathrm{C}^{3, \alpha}\left(\overline{\mathrm{V}}_{n}\right)$ et n'est pas nulle car $h$ ne l'est pas.

Remarque. Dans le cas particulier de l'équation (1) sur les ouverts bornés de $\mathbb{R}^{n}$ et lorsque la fonction $f$ est constante, la méthode de réflexion développée par Nirenberg [6] et utilisée dans [5], donne les estimées nécessaires pour empêcher toute concentration de la suite $w_{p}$. Dans le cas plus général étudié ici, nous avons seulement, pour l'instant, montré l'inégalité:

$$
\int_{\mathrm{V}_{n}} f\left|w_{0}+h\right|^{N} \leq \liminf _{p \rightarrow N} \int_{\mathrm{V}_{n}} f\left|w_{p}+h\right|^{p}=\gamma
$$

Des hypothèses géométriques nous permettront d'éliminer les phénomènes de concentration, ce qui nous donnera l'égalité dans la relation (17).

\section{Concentration-Minimisation}

Cette partie concerne l'analyse de la concentration qui peut avoir lieu lorsque l'exposant $\mathrm{p}$ tend vers l'exposant critique $N$. Rappelons la définition suivante $[12]:$

Définition 1. Un point $Q$ est dit de concentration pour la suite $\left(\phi_{p}\right)$ avec $\phi_{p}=$ $w_{p}+h$ si

$$
\exists \epsilon>0 \text { tel que } \forall \delta>0 \lim _{p \rightarrow N} \int_{\mathrm{B}_{Q}(\delta)}\left|\phi_{p}\right|^{p}>\epsilon .
$$

Supposons qu'il existe un point de concentration P. Dans ce qui suit, suivant que $\mathrm{P}$ est sur le bord ou à l'intérieur de $\mathrm{V}_{n}$, on met en évidence une condition qui amène à une contradiction. Nous allons montrer la proposition suivante:

Proposition 1. Soit $w_{0} \neq 0$ la limite faible de la suite minimisante $w_{p}$ obtenue quand $p$ tend vers $N$. Posons $\mu(\gamma)=\lim _{p \rightarrow N} \mu_{p, \gamma}$. K désigne la meilleure constante dans l'inclusion de Sobolev $H_{1}$ dans $\mathrm{L}_{N}$. Si les deux conditions suivantes sont vérifiées

$$
\mathrm{K}^{2} \mu(\gamma)(\sup f)^{2 / N} \frac{(\gamma)^{1-2 / N}}{\gamma-\int_{\mathrm{V}_{n}} f\left|w_{0}+h\right|^{N-2}\left(w_{0}+h\right) h} \quad<1
$$




$$
\begin{gathered}
\frac{\mathrm{K}^{2}}{\gamma-\int f\left|w_{0}+h\right|^{N-2}\left(w_{0}+h\right) h} \mu(\gamma)(\sup f)^{2 / N} \times \\
\left(\gamma+\mathrm{C}_{1} \int_{\mathrm{V}_{n}} h^{N}+\mathrm{C}_{2} \int_{\mathrm{V}_{n}} w_{0}{ }^{N-1}|h|\right)^{1-2 / N}<1 \\
\text { dite condition au bord, }
\end{gathered}
$$

où $\mathrm{C}_{1}$ et $\mathrm{C}_{2}$ sont deux constantes indépendantes de $p$, alors la suite des minimiseurs $w_{p}$ n'a pas de point de concentration et la limite $w_{0}$ est une solution minimisante pour la fonctionnelle d'énergie $\mathrm{I}(u)=\int|\nabla u|^{2}+\overline{\mathrm{R}} u^{2}$ sur l'ensemble $\mathrm{A}_{N, \gamma}$.

La preuve est composée de plusieurs étapes : le passage à la limite quand il n'y a pas concentration, l'expression des conditions de non-concentration et l'impossibilité de concentration sous les conditions.

Preuve. Quand l'exposant reste sous-critique la convergence de $\left(w_{p}\right)$ est forte, donc

$$
\lim _{p \rightarrow N} \int_{\mathrm{V}_{n}} f\left|w_{p}+h\right|^{p-2}\left(w_{p}+h\right) h=\int_{\mathrm{V}_{n}} f\left|w_{0}+h\right|^{N-2}\left(w_{0}+h\right) h
$$

Il en est de même lorsque l'exposant est critique et qu'il n'y a pas de phénomène de concentration. Il en résulte

$$
\lim _{p \rightarrow N} \gamma-\int_{\mathrm{V}_{n}} f\left|w_{p}+h\right|^{p}=\gamma-\int_{\mathrm{V}_{n}} f\left|w_{0}+h\right|^{N}
$$

Les conditions de la proposition sont obtenues de la manière suivante:

\subsubsection{Point de concentration dans $\stackrel{o}{\mathrm{~V}_{n}}$}

Supposons que $\mathrm{P}$ soit un point de concentration dans $\stackrel{o}{\mathrm{~V}}_{n}$. Soit $\eta \in \mathrm{C}^{\infty}\left(\mathrm{V}_{n}\right)$ une fonction à support compact dans une boule $\mathrm{B}_{\mathrm{P}}(\delta)$, égale à 1 sur une boule $\left.\mathrm{B}_{(} \delta / 2\right)(\mathrm{P})$. En multipliant l'équation (10) par $\eta^{2}\left|w_{p}+h\right|^{k-1}\left(w_{p}+h\right), k>1$ puis en intégrant, on obtient:

quel que soit $\epsilon>0$ il existe $\mathrm{C}_{\epsilon}$ tel que:

$$
\begin{gathered}
\left(\frac{4 k}{(k+1)^{2}}-\frac{2(k-1) \epsilon}{(k+1)^{2}}\right) \int_{\mathrm{B}_{\mathrm{P}}(\delta)}\left|\nabla \eta \phi_{p}^{(k+1) / 2}\right|^{2} \leq \mathrm{C}_{\epsilon} \int_{\mathrm{B}_{\mathrm{P}}(\delta)}\left|\phi_{p}\right|^{k+1}+ \\
\lambda_{p, \gamma} \sup f^{2 / p}\left|\eta \phi_{p}^{(k+1) / 2}\right|_{p}^{2}\left(\int_{\mathrm{B}_{\mathrm{P}}(\delta)} f\left|\phi_{p}\right|^{p}\right)^{1-2 / p}
\end{gathered}
$$

où l'on a posé $\phi_{p}=w_{p}+h$. Or comme

$$
\lambda_{p, \gamma}=\frac{\mu_{p, \gamma}}{\int_{\mathrm{V}_{n}}\left|w_{p}+h\right|^{p-2}\left(w_{p}+h\right) w_{p}}
$$

en utilisant l'inégalité de Sobolev classique avec les meilleures constantes, (voir [1]) on obtient pour toute fonction $u$ dans $H_{1}$ :

$$
\|u\|_{\mathrm{L}_{N}}^{2} \leq \mathrm{K}^{2}\|\nabla u\|_{\mathrm{L}_{2}}^{2}+\mathrm{A}\|u\|_{\mathrm{L}_{2}}^{2}
$$


$\mathrm{K}$ représente la meilleure constante dans les inclusions de Sobolev ( $\mathrm{K}$ ne dépend que de la dimension $n$ ). On obtient, en posant $u=\eta \phi_{p}^{(k+1) / 2}$,

$$
\left\|\eta \phi_{p}^{(k+1) / 2}\right\|_{p}^{2} \leq \operatorname{Vol}_{g}\left(\mathrm{~V}_{n}\right)^{\beta}\left(\mathrm{K}^{2}\left\|\nabla\left(\eta \phi_{p}^{(k+1) / 2}\right)\right\|_{2}^{2}+\mathrm{A}\left\|\eta \phi_{p}^{(k+1) / 2}\right\|_{2}^{2}\right.
$$

Ici, $\beta=2\left(\frac{1}{p}-\frac{1}{N}\right)$. En combinant ces deux dernières relations et en tenant compte du fait que $\left(\int_{\mathrm{B}_{\mathrm{P}}(\delta)} f\left|\phi_{p}^{p}\right|\right)^{1-2 / p}=\left(\gamma-\int_{\mathrm{V}_{n}-\mathrm{B}_{\mathrm{P}}(\delta)} f\left|w_{p}+h\right|^{p}\right)^{1-2 / p}$, il s'ensuit que si

$$
\lim _{p \rightarrow N} \frac{\mathrm{K}^{2} \sup f^{2 / p} \mu_{p, \gamma}}{\int_{\mathrm{V}_{n}}\left|w_{p}+h\right|^{p-2}\left(w_{p}+h\right) w_{p}}\left(\gamma-\int_{\mathrm{V}_{n}-\mathrm{B}_{\mathrm{P}}(\delta)} f\left|w_{p}+h\right|^{p}\right)^{1-2 / p}<1
$$

alors

$$
\left\|\eta w_{p}^{(k+1) / 2}\right\|_{p}^{2} \text { et }\left\|\nabla\left(\eta w_{p}^{(k+1) / 2}\right)\right\|_{2}^{2}
$$

sont bornées pour $\mathrm{k}$ voisin de 1 , donc aussi

$$
\int_{\mathrm{B}_{\mathrm{P}}(\delta / 2)} \eta^{N}\left|w_{p}\right|^{n(k+1) /(n-2)}
$$

ceci en utilisant les injections continues de Sobolev.

\subsubsection{Point de concentration sur $\partial \mathrm{V}_{n}$}

Supposons que $\mathrm{P}$ soit un point de concentration sur $\partial \mathrm{V}_{n}$. Comme dans la partie précédente on cherche à obtenir des estimées locales sur la norme $\mathrm{L}^{p}$ de la solution $w_{p}$, dans une boule centrée en $\mathrm{P}$ de rayon petit.

Soit $\eta \in \mathrm{C}^{\infty}$ une fonction définie par

$$
\begin{gathered}
\eta=1 \text { sur } \quad \mathrm{B}_{\mathrm{P}}(\delta / 2) \\
\eta=0 \text { sur } \quad \mathrm{V}_{n}-\mathrm{B}_{\mathrm{P}}(\delta)
\end{gathered}
$$

Comme $\eta$ n'est pas nulle sur $\partial \mathrm{B}_{\mathrm{P}}(\delta)$, contrairement au cas précédent, on multiplie cette fois-ci l'équation:

$$
\Delta w_{p}+\overline{\mathrm{R}} w_{p}=\lambda_{p, \gamma} f\left|w_{p}+h\right|^{p-2}\left(w_{p}+h\right)
$$

par la fonction $\eta^{2}\left|w_{p}\right|^{k-1} w_{p}$ avec $k>1$. On notera $\mathrm{B}_{\mathrm{P}}^{+}$la demi-boule riemannienne centrée au point $\mathrm{P}$ de rayon petit.

Comme dans le paragraphe précédent, on obtient après calculs:

$$
\begin{gathered}
\left(\frac{4 k}{(k+1)^{2}}-\frac{2(k-1) \epsilon}{(k+1)^{2}}\right) \int_{\mathrm{B}_{\mathrm{P}}^{+}}\left(\nabla \eta w_{p}^{(k+1) / 2}\right)^{2} \leq \mathrm{C}_{\epsilon} \int_{\mathrm{B}_{\mathrm{P}}^{+}}\left|w_{p}\right|^{k+1}+ \\
\lambda_{p, \gamma}\left(\int_{\mathrm{B}_{\mathrm{P}}^{+}} f \eta^{2}\left|w_{p}\right|^{k}\left|w_{p}+h\right|^{p-2}\left(w_{p}+h\right)\right)
\end{gathered}
$$

En utilisant l'inégalité suivante:

$$
\forall x, y,|x+y|^{p} \leq \mathrm{C}_{p}\left(|y|^{p}+|x|^{p}\right)
$$

le second membre de (32) devient: 


$$
\int_{\mathrm{B}_{\mathrm{P}}^{+}}\left|w_{p}\right|^{k+1}+\mathrm{C} \lambda_{p, \gamma} \int_{\mathrm{B}_{\mathrm{P}}^{+}} \eta^{2}\left|w_{p}\right|^{k} f|h|^{p-1}+\lambda_{p, \gamma}\left(\int_{\mathrm{B}_{\mathrm{P}}^{+}} f \eta^{2}\left|w_{p}\right|^{k+p-1}\right)
$$

Lorsque $\mathrm{p}$ tend vers $\mathrm{N}, \lambda_{p, \gamma}$ et $\int_{\mathrm{B}_{\mathrm{P}}^{+}} f\left|w_{p}\right|^{k}$ sont bornés. On étudie alors le dernier terme de (34). En utilisant l'identité de Hölder et l'expression du multiplicateur de Lagrange $\lambda_{p, \gamma}$, on obtient:

$$
\begin{aligned}
\lambda_{p, \gamma}\left(\int_{\mathrm{B}_{\mathrm{P}}^{+}} f \eta^{2}\left|w_{p}\right|^{k+p-1}\right) & \leq \frac{\mu_{p, \gamma} f(\mathrm{P})^{2 / p}}{\gamma-\int f\left|w_{p}+h\right|^{p-2}\left(w_{p}+h\right) h}\left|\eta w_{p}^{(k+1) / 2}\right|_{p}^{2} \times \\
& \left(\left.\int_{\mathrm{B}_{\mathrm{P}}^{+}} f|w|\right|_{p} ^{p}\right)^{1-2 / p}
\end{aligned}
$$

En utilisant les inégalités de Sobolev et la majoration $|x|^{p} \leq|x+h|^{p}+\mathrm{C}_{1}|h|^{p}+$ $\mathrm{C}_{2}|x|^{p-1}|h|$, on a

$$
\int_{\mathrm{B}_{\mathrm{P}}^{+}} f|w|_{p}^{p} \leq \int_{\mathrm{B}_{\mathrm{P}}^{+}} f\left|w_{p}+h\right|^{p}+\mathrm{C}_{1} \int_{\mathrm{B}_{\mathrm{P}}^{+}}|h|^{p}+\mathrm{C}_{2} \int_{\mathrm{B}_{\mathrm{P}}^{+}}\left|w_{p}\right|^{p-1}|h|
$$

où $\mathrm{C}_{1}$ et $\mathrm{C}_{2}$ sont deux constantes positives, indépendantes de $\mathrm{p}$ (pour $\mathrm{p}$ proche de $N$ ). On peut maintenant identifier une condition pour qu'il n'y ait pas de point de concentration sur $\partial \mathrm{V}_{n}$ pour la suite $w_{p}\left(\right.$ ou $\left.w_{p}+h\right)$ :

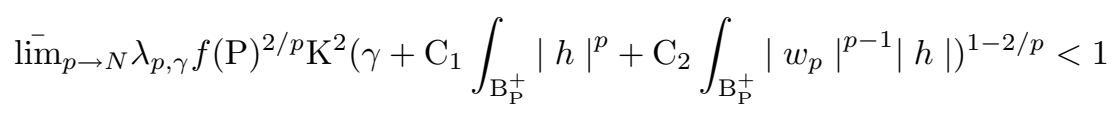

soit en passant à la limite

$$
\begin{gathered}
\frac{\mathrm{K}^{2}}{\gamma-\int f\left|w_{0}+h\right|^{N-2}\left(w_{0}+h\right) h} \mu(\gamma) f(\mathrm{P})^{2 / N} \times \\
\left(\gamma+\mathrm{C}_{1} \int_{\mathrm{V}_{n}}|h|^{N}+\mathrm{C}_{2} \int_{\mathrm{V}_{n}}\left|w_{0}\right|^{N-1}|h|\right)^{1-2 / N}<1
\end{gathered}
$$

\subsubsection{Non concentration}

Montrons qu'il n'y a pas de point de concentration lorsque les quantités (28) sont bornées. Supposons que P soit un point de concentration. En utilisant la définition (18) de concentration, l'inégalité de Hölder permet d'obtenir la majoration suivante

$$
\begin{aligned}
\epsilon< & \int_{\mathrm{B}_{\mathrm{P}}(\delta)}\left|w_{p}+h\right|^{p} \leq \mathrm{C}\left(\int_{\mathrm{B}_{\mathrm{P}}(\delta)}\left|w_{p}+h\right|^{N}\right)^{p / N}= \\
& \mathrm{C}\left(\int_{\mathrm{B}_{\mathrm{P}}(\delta)}\left|w_{p}+h\right|^{N-1}\left|w_{p}+h\right|\right)^{p / N} \\
\leq & \left\{\int_{\mathrm{BP}_{\mathrm{P}}(\delta)}\left|w_{p}+h\right|^{n(k+1) /(n-2)}\right\}^{\frac{p(n+2)}{n N(k+1)}} \times \\
& \left\{\int_{\mathrm{B}_{\mathrm{P}}(\delta)}\left|w_{p}+h\right|^{n(k+1) /(k n-2)}\right\}^{\frac{p(n k-2)}{n N(k+1)}}
\end{aligned}
$$

C désigne une constante indépendante de p. D'où en utilisant (29)

$$
\epsilon<\int_{\mathrm{B}_{\mathrm{P}}(\delta)}\left|w_{p}+h\right|^{p} \leq \mathrm{C}\left(\int_{\mathrm{B}_{\mathrm{P}}(\delta)}\left|w_{p}+h\right|^{k_{1}}\right)^{p / k_{1}}
$$


avec $k_{1}=n(k+1) /(k n-2)<N$ puisque $k>1$. Alors pour $p>k_{1}$, (p tend vers $\mathrm{N}$ )

$$
\begin{gathered}
\int_{\mathrm{BP}_{\mathrm{P}}(\delta)}\left|w_{p}+h\right|^{k_{1}} \leq \mathrm{Cte}\left(\int_{\mathrm{B}_{\mathrm{P}}(\delta)} f\left|w_{p}+h\right|^{p}\right)^{k_{1} / p} \operatorname{Vol}_{g}^{1-k_{1} / p}\left(\mathrm{~B}_{\mathrm{P}}(\delta)\right) \leq \\
\mathrm{C} \gamma^{k_{1} / p} \delta^{n\left(1-k_{1} / p\right)}
\end{gathered}
$$

Le terme de droite de (41) est rendu arbitrairement petit par le choix de $\delta$, ce qui est en contradiction avec la définition de la concentration, puisque $\epsilon>0$ ne dépend pas de $\delta$.

\subsection{Convergence des minima $\mu_{p, \gamma}$}

Une minoration du minimum d'énergie est utilisée maintenant pour obtenir la limite de $\mu_{p, \gamma}$ quand $\mathrm{p}$ tend vers $\mathrm{N}$. On note $\mu(\gamma)=\mu(\gamma, N)$.

Lemme 3. Il existe une suite $\gamma_{p} \rightarrow \gamma$ telle que $\lim _{p \rightarrow N} \mu_{p, \gamma_{p}} \leq \mu(\gamma)$

Preuve. Soit $\epsilon>0$, il existe une fonction $\eta \in \mathrm{A}_{N, \gamma}$ telle que :

$$
\mu(\gamma) \leq \int|\nabla \eta|^{2}+\int \overline{\mathrm{R}} \eta^{2} \leq \mu(\gamma)+\epsilon
$$

Posons $\gamma_{p}=\int f|\eta+h|^{p}$. Comme

$$
\lim _{p \rightarrow N} \int f|\eta+h|^{p}=\int f|\eta+h|^{N}=\gamma>\int f|h|^{N}
$$

$\gamma_{p}>\int f|h|^{N}$ pour p proche de $N$. La contrainte sur $\gamma$ est donc vérifiée pour les suites sous-critiques. Soit une suite de minimiseurs $w_{p} \in \mathrm{A}_{p, \gamma_{p}}$. Par hypothèse,

$$
\mu_{p, \gamma_{p}}=\int\left|\nabla w_{p}\right|^{2}+\int \mathrm{R} w_{p}^{2} \leq \int|\nabla \eta|^{2}+\int \overline{\mathrm{R}} \eta^{2}
$$

Donc $\lim \mu_{p, \gamma_{p}} \leq \mu(\gamma)$. Le lemme 3 sera alors utilisé pour l'étude de la convergence de la suite sous-critique, puisqu'il est maintenant possible de choisir des solutions dans l'ensemble $\mathrm{A}_{p, \gamma_{p}}$, qui convergeront vers la solution voulue.

\section{Conditions géométriques et fonctions tests}

L'objet de cette partie est de montrer que sous les hypothèses du théorème (1), les deux conditions de la proposition énoncée précédemment sont vérifiées lorsque l'on a choisi un paramètre $\gamma$ suffisamment grand.

Pour montrer que la première condition est vérifiée, deux quantités vont être évaluées, $\gamma_{\epsilon}=\int_{\mathrm{V}_{n}} f\left|u_{\epsilon}+h\right|^{N}$ et $\mu_{\epsilon}=\int\left|\nabla u_{\epsilon}\right|^{2}+\overline{\mathrm{R}} u_{\epsilon}^{2}$, en utilisant les fonctions 
de $\stackrel{o}{H_{1}}\left(\mathrm{~V}_{n}\right)$ suivantes:

$$
\begin{aligned}
& u_{\epsilon}(r)=\left(\frac{1}{\left(r^{2}+\epsilon^{2}\right)^{(n-2) / 2}}-\frac{1}{\left(\delta^{2}+\epsilon^{2}\right)^{(n-2) / 2}}\right) \quad \text { sur } \mathrm{B}_{\mathrm{P}}(\delta) \\
& u_{\epsilon}(\mathrm{P})=\quad 0 \quad \text { sur } \mathrm{V}_{n}-\mathrm{B}_{\mathrm{P}}(\delta)
\end{aligned}
$$

où $r$ représente la distance géodésique au point $\mathrm{P}$. Ces fonctions introduites dans [2], sont radiales en $\mathrm{P}$, nulles en dehors de la boule de rayon $\delta, \delta$ étant choisi plus petit que le rayon d'injectivité. Ces deux quantités $\gamma_{\epsilon}$ et $\mu_{\epsilon}$ serviront à montrer que le quotient $Q_{\epsilon}$ :

$$
Q_{\epsilon}=(\sup f)^{2 / N} \mathrm{~K}^{2} \mu_{\epsilon} \frac{\gamma_{\epsilon}{ }^{1-2 / N}}{\gamma_{\epsilon}-\int_{\mathrm{V}_{n}} f\left|w_{0}+h\right|^{N-2}\left(w_{0}+h\right) h}
$$

est strictement plus petit que 1 .

\subsection{Evaluation du quotient $Q_{\epsilon}$}

Dans ce paragraphe, on etudie le développement asymptotique de $\gamma_{\epsilon}$ et $\mu_{\epsilon}$. Dans la suite, $d \Omega$ désigne l'élément de volume de la sphère $S^{n-1}(1)$,

$$
\gamma_{\epsilon}=\int_{\mathrm{V}_{n}} f\left|u_{\epsilon}+h\right|^{N}=\int_{0}^{\delta} r^{n-1} d r \int_{\mathrm{S}(r)} f\left|u_{\epsilon}+h(x)\right|^{N} d \Omega
$$

Posons $x=\epsilon u$

$$
\gamma_{\epsilon}=\int_{0}^{\delta / \epsilon} \epsilon^{n} u^{n-1} d u \int_{\mathrm{S}(r)} f(\epsilon u) \sqrt{g(\epsilon u)} \epsilon^{-2 n}\left|v+\epsilon^{n-2} h(\epsilon u)\right|^{N} d \Omega
$$

où

$$
\begin{aligned}
v(r) & =\left(\frac{1}{\left(r^{2}+1\right)^{(n-2) / 2}}-\frac{\epsilon^{n-2}}{\left(\delta^{2}+\epsilon^{2}\right)^{(n-2) / 2}}\right) & & \text { sur } \mathrm{B}_{\mathrm{P}}(\delta) \\
& = & & \text { sur } \mathrm{V}_{n}-\mathrm{B}_{\mathrm{P}}(\delta)
\end{aligned}
$$

Pour évaluer $\gamma_{\epsilon}$, on étudie plus précisement le développement de

$$
\mathrm{G}(u)=\int_{\mathrm{S}(r)} f(\epsilon u) \sqrt{g(\epsilon u)} \epsilon^{-2 n}\left|v+\epsilon^{n-2} h(\epsilon u)\right|^{N} d \Omega .
$$

En utilisant le développement de Taylor suivant

$$
\begin{gathered}
\left|v+\epsilon^{n-2} h(\epsilon u)\right|^{N}=|v|^{N}+ \\
\epsilon^{n-2} \int_{0}^{1} N\left|v+t \epsilon^{n-2} h(\epsilon u)\right|^{N-2}\left(v+t \epsilon^{n-2} h(\epsilon u)\right) h(\epsilon u) d t
\end{gathered}
$$

on obtient

$$
\mathrm{G}(u)=\int_{\mathrm{S}(r)} f(\epsilon u) \sqrt{g(\epsilon u)} \epsilon^{-2 n}|v|^{N} d \Omega+\mathrm{R}_{\epsilon}(u)
$$


avec

$$
\begin{gathered}
\mathrm{R}_{\epsilon}(u)=\epsilon^{-n-2} \int_{\mathrm{S}(r)} f(\epsilon u) \sqrt{g(\epsilon u)} \int_{0}^{1} N \\
\left|v+t \epsilon^{n-2} h(\epsilon u)\right|^{N-2}\left(v+t \epsilon^{n-2} h(\epsilon u)\right) h(\epsilon u) d t d \Omega
\end{gathered}
$$

puis

$$
\mathrm{R}_{\epsilon}(\gamma)=\int_{0}^{\delta / \epsilon} \epsilon^{n} u^{n-1} d u \mathrm{R}_{\epsilon}(u)
$$

Alors

$$
\mathrm{R}_{\epsilon}(\gamma) \leq \mathrm{C} \epsilon^{-2} \int_{0}^{\delta / \epsilon} u^{n-1} d u \int_{\mathrm{S}(u)}|f|\left|v+\epsilon^{n-2}\right| \sup h \|^{N-1}|\sup h| d \Omega
$$

C est ici une constante qui ne dépend pas de $\epsilon$ et qui majore $\sqrt{|g|}$. Ainsi en posant $\hat{\gamma}_{\epsilon}=\int_{\mathrm{V}_{n}} f u_{\epsilon}{ }^{N}$, et en revenant à la définition de $\gamma_{\epsilon}$, on obtient,

$$
\gamma_{\epsilon}=\hat{\gamma}_{\epsilon}+\mathrm{R}_{\epsilon}(\gamma)
$$

Or $\hat{\gamma}_{\epsilon}=0\left(\epsilon^{-n}\right)$ car

$$
\hat{\gamma}_{\epsilon}=\epsilon^{-n} \int_{0}^{\delta / \epsilon} r^{n-1} d r \int_{\mathrm{S}(r \epsilon)} f(\epsilon x) v_{\epsilon}^{N} \leq \mathrm{C} \epsilon^{-n} \int_{0}^{\delta / \epsilon} r^{n-1} d r v_{\epsilon}^{N}
$$

De plus $\int_{0}^{\delta / \epsilon} r^{n-1} d r v_{\epsilon}^{N} \leq \mathrm{C}$ puisque $v_{\epsilon}^{N} \leq v_{\epsilon=0}^{N}$ est intégrable. De même pour le reste $\mathrm{R}_{\epsilon}(u)$ qui est intégrable.

$$
\mathrm{R}_{\epsilon}(\gamma) \leq \epsilon^{-2} \mathrm{C} \int_{0}^{\delta / \epsilon} u^{n-1} d u\left|v_{\epsilon}+\epsilon^{n-2}\right| \sup h||^{N-1} \leq \mathrm{C} \epsilon^{-2}
$$

Ainsi

$$
\gamma_{\epsilon}=\hat{\gamma}_{\epsilon}\left(1+\mathrm{O}\left(\epsilon^{n-2}\right)\right)
$$

\subsection{Conditions géométriques}

Compte tenu de l'hypothèse faite sur la non nullité du tenseur de Weyl en un point, seuls les termes jusqu'à l'ordre $\epsilon^{4}$ auront de l'intérêt. Si l'on pose

$$
\mathrm{S}_{\epsilon}=(\sup f)^{2 / N} \mathrm{~K}^{2} \mu_{\epsilon} \hat{\gamma}\left(u_{\epsilon}\right)^{-2 / N}
$$

en dimension plus grande que 6 et en utilisant l'estimation (61) sur $\gamma_{\epsilon}$ on obtient $Q_{\epsilon}=\mathrm{S}_{\epsilon}+o\left(\epsilon^{4}\right)$. Les calculs explicites de $\mathrm{S}_{\epsilon}$ ont été effectués dans [2], on pourra s'y référer. Donnons simplement les résultats:

$$
Q_{\epsilon}=Q_{0}+Q_{1} \epsilon+Q_{2} \epsilon^{2}+Q_{3} \epsilon^{3}+Q_{4} \epsilon^{4}+o\left(\epsilon^{4}\right)
$$

Les calculs sur les termes du premier ordre montrent que $Q_{0}=1$, puis $Q_{1}=$ $Q_{3}=0$ puisque il n'y a pas de terme d'ordre impair. Enfin, $Q_{2}=0$ grâce à 
l'hypothèse $\Delta_{g} f(\mathrm{P})=0$. De plus:

$$
\begin{gathered}
Q_{4}=\mathrm{T} \frac{(n+2) 10}{(n-4)(n-6)}-\left(\frac{\mathrm{R}^{2}}{6 n}+\frac{\Delta \mathrm{R}}{6 n}\right) \frac{1}{2 n(n-4)(n-6)}-\frac{(n+2)}{(n-4)}\left(\frac{\Delta^{2} f}{4 ! n(n+2)}+\right. \\
\left.\frac{n-1}{36 n^{2}(n-2)} \mathrm{R}^{2}\right)
\end{gathered}
$$

avec

$$
\mathrm{T}=\frac{1}{(n+2) n 4 !}\left(\frac{6 \Delta \mathrm{R}}{5}-\frac{2 \mathrm{R}^{i j} \mathrm{R}_{i j}}{15}-\frac{\mathrm{R}_{i j k l}^{2}}{5}+\frac{2 \mathrm{R}^{i j} \mathrm{R}_{i j}}{3}+\frac{\mathrm{R}^{2}}{3}\right)
$$

Les hypothèses ont été choisies pour que $Q_{4}$ soit non nul. Pour simplifier ce terme, remarquons qu'il existe une métrique conforme à la métrique initiale $g^{\prime}=e^{\psi} g$, telle que $\mathrm{R}\left(g^{\prime}\right)(\mathrm{P})=0$ et $\mathrm{R}_{i j}\left(g^{\prime}\right)=0$ [2]. $\psi$ est choisie nulle en dehors d'une boule de centre P. L'invariance conforme de la fonctionnelle I montre alors que les calculs précédents faits en utilisant $\frac{u_{\epsilon}}{e^{\psi}}$ comme fonctions tests au lieu des $u_{\epsilon}$ donnent les mêmes résultats.

Remarquons de plus que les conditions sur $f$ au point du maximum sont bien invariantes par changement de métrique conforme: au maximum de f, $\Delta_{g} f(\mathrm{P})=$ $0=\Delta_{g^{\prime}} f(\mathrm{P})$ et comme les dérivées d'ordre plus petit que 3 en $\mathrm{P}$ sont nulles, $\Delta_{g}^{2} f=0=\Delta_{g^{\prime}}^{2} f$. L'invariant conforme du tenseur de Weyl W $(g)$ nous permet alors d'écrire

$$
Q_{4}=\frac{1}{12 n(n-4)(n-6)}\left(-e^{\psi}\left|\mathrm{W}^{2}(g)\right|_{g}\right)-\frac{\Delta_{g^{\prime}}^{2} f(\mathrm{P})}{4 ! n(n-4)}
$$

Finalement lorsque $\Delta_{g^{\prime}}^{2} f=0$, on a bien $Q_{4}<0$ et $Q_{\epsilon}<1$.

Remarque 1. Lorsque $\Delta_{g^{\prime}}^{2} f$ reste petit devant le module du tenseur de Weyl, la proposition reste valable, mais elle ne se quantifie pas aisément en termes géométriques.

La condition de bord de la proposition 1 est satisfaite de la même façon. En effet les termes où apparaissent les constantes $\mathrm{C}_{1}$ et $\mathrm{C}_{2}$ sont bornés, ils sont donc négligeables devant le terme en $\gamma_{\epsilon}=\mathrm{O}\left(\epsilon^{2-n}\right)$. La condition de non concentration au bord est encore vérifiée grâce à la non nullité du tenseur de Weyl, au point $\mathrm{P}$.

Remarque 2. Quand le tenseur de Weyl est nul au point P, il faut alors pousser plus loin les développements. Les conclusions sont les mêmes tant que le seuil de l'exposant du développement limité n'excède pas n-2. Dans le cas contraire, c'est la masse positive (voir [11]) qui apparaît avant le terme contenant le tenseur de Weyl W. Ce cas a été traité dans [10].

\subsubsection{Quand $a \neq \overline{\mathrm{R}}$}

Pour montrer que le quotient $Q_{\epsilon}$ est plus petit que 1, on utilise la non nullité du terme d'ordre 2 dans le développement qui provient de l'inégalité:

$$
c_{n} a(\mathrm{P})+\frac{(n-4) \Delta f(\mathrm{P})}{2 f(\mathrm{P})}<\mathrm{R}(\mathrm{P})
$$


En imposant les mêmes conditions sur $f$ que celles énoncées au théorème 1, la condition (67) au point $\mathrm{P}$ s'écrit $a(\mathrm{P})<\overline{\mathrm{R}}$. Dans ce cas, le théorème est vrai à partir de la dimension 5 .

\section{Questions ouvertes}

- Trouver le plus petit $\gamma$ tel que la condition de non-concentration soit vérifiée.

- Lorsque le maximum de f est atteint uniquement sur le bord, comment satisfaire la condition de non-concentration?

- Que dire des cas où la dimension est inférieure à 6 , lorsque la variété n'est pas localement conformément plate?

Terminons par une remarque concernant la convergence d'une famille de solutions de l'équation (1), lorsque la donnée au bord est une suite de fonctions qui tend uniformément vers 0 . En fait, il n'est pas possible par cette méthode d'obtenir des solutions nodales nulles sur le bord car lorsque le phénomène de concentration est évité, les seules solutions minimisantes nulles au bord sont positives. On pourra se reporter à [10].

\section{References}

[1] T. Aubin, Some Nonlinear problems in Riemannian Geometry. Berlin Springer-Verlag, 1998

[2] T. Aubin, Equations differentielles non linéaires et problème de Yamabe concernant la courbure scalaire, J. Math. pures et appl. 55 (1976), 269-296.

[3] T. Aubin, Meilleures constantes dans le théorème d'inclusion de Sobolev et un théorème de Fredholm non linéaire pour la transformation conforme de la courbure scalaire $J$. Funct. Anal. 32 (1979), 148-174.

[4] H. Brezis, On some variational problem with limiting Sobolev exponent. Progress in variational methods in Hamiltonian systems and elliptic equations (L'Aquila, 1990) Pitman Res. Notes Math. Ser. 243, Longman Sci. Tech., Harlow (1992), 42-51.

[5] L. Caffareli, J. Spruck, Variational Problems with Critical Sobolev Growth and positive Dirichlet Data Indiana Vol. 39 No. 1 (1990), 1-18.

[6] Gidas Ni Nirenberg, Symetry and Related Properties via the Maximum Principle. Comm. Math. Phys. 68 (1979), 209-243.

[7] D. Gilbard, N. Trudinger, Elliptical partial differential equations of second order, Springer Verlag 1983

[8] E. Hebey, M. Vaugon, Existence and multiplicity of Nodal Solutions for Nonlinear Elliptic Equations with Critical Sobolev Growth J. Funct. Anal. 119 No. 2 (1994), 298-318.

[9] D. Holcman, Solutions nodales sur les variétés riemanniennes à bord. (French) [Nodal solutions on Riemannian manifolds with boundary] C. R. Acad. Sci. Paris Sér. I Math. 326 (1998), no. 11, 1321-1324.

[10] D. Holcman, Thèse de doctorat de l'université Paris VI 1998.

[11] J. Lee, T.H. Parker, The Yamabe problem. Bull of A.M.S. 17 No. 1 (1987), 37-91.

[12] M. Vaugon, Transformation de la courbure scalaire sur une variété Riemannienne compacte J. of Funct. Anal. 71 No. 1 (1987), 182-194. 
David Holcman

Université Pierre et Marie Curie, Paris VI

tour 46-00

case postale 247

4 place Jussieu

F-75252 Paris cedex 05

France

e-mail : holcman@math.jussieu.fr

(Received: February 29, 2000) 